\title{
Comparison of Leukotriene Receptor Antagonists and Anti-Histamines as an Add-On Therapy in Patients with Asthma Complicated by Allergic Rhinitis
}

\author{
MUNETSUGU NISHIMURA, TAKEHARU KOGA, TOMOKO KAMIMURA, RUMI SATO, \\ HIROKI NATORI*, KAZUHIKO MATSUO* AND HISAMICHI AIZAWA* \\ Department of Respiratory Medicine, Asakura Medical Association Hospital, Asakura 838-0069 and \\ *Department of Internal Medicine, Kurume University School of Medicine, \\ Kurume 830-0011, Japan
}

Received 25 August 2010, accepted 24 November 2010

\author{
Edited by TADASHI NAKASHIMA
}

\begin{abstract}
Summary: Patients with asthma are often complicated by allergic rhinitis, and the intimate pathophysiological association between allergic rhinitis and asthma often imposes a significant morbidity on affected individuals. The present study was conducted to assess the clinical efficacies of leukotriene receptor antagonists (LTRAs) and anti-histamines on asthma as an add-on therapy in patients with asthma complicated by allergic rhinitis. Consecutive patients with asthma were recruited to fill in systematic self-administered questionnaires concerning symptoms and conditions related to asthma and allergic rhinitis. The questionnaire was conducted twice, one month apart, and the attending physicians gave detailed information on disease control and medications on both occasions. In the study 3,140 patients with asthma participated, and 634 had concomitant allergic rhinitis (mean age: 53.1, 389 female). The second survey disclosed that treatment with LTRAs or anti-histamines had been added in 26 patients and 19 patients, respectively, without any changes in other medications. There were no significant differences in age, gender, severity of disease, or baseline treatments. The initial survey indicated that the patients who were treated with LTRAs had significantly more severe asthma-related symptoms (i.e. wheeze, cough and sleep disturbance) and experienced greater dissatisfaction with the treatment than did those who were treated with anti-histamines. The second survey disclosed significant reductions in sneezing $(p=0.03)$, rhinorrhea $(p=0.01)$, dyspnea $(p=0.046)$, sleep disturbance $(p=0.02)$, over-all asthma symptoms $(p=0.013)$, and an improvement in satisfaction with treatment $(p=0.019)$ in patients to whom LTRAs were added-on, whereas the patients receiving anti-histamines reported no significant changes in these symptoms. These results suggest that LTRAs are more effective than anti-histamines as an add-on therapy in symptomatic patients with asthma complicated by allergic rhinitis.
\end{abstract}

Key words allergic rhinitis, asthma, anti-histamine, leukotriene receptor antagonist, add-on therapy

\section{INTRODUCTION}

The association between asthma and allergic rhinitis has been gaining attention as part of a continuum of airway pathology, as these two disorders not only coexist frequently but also have intimate clinical and pathological associations. For instance, clinical observations have documented that asthma attacks are coincident with a worsening of nasal symptoms [1], and that cough in patients with asthma complicated by allergic rhinitis is more severe than that in patients with asthma alone [2]. A shared pathophysiology of

Correspondence to: Dr. Takeharu Koga, Department of Respiratory Medicine, Asakura Medical Association Hospital, Raiharu 422-1, Asakura 838-0069, Japan. Tel:+81-946-23-0077 Fax:+81-946-23-0076 E-mail:koga.tk@asakura-med.or.jp 
the two disorders is illustrated by the existence of a significant correlation between the number of nasal eosinophils and forced expiratory volume in one sec [3], and by an exaggerated responsiveness of the lower airways induced by allergic nasal challenge [4] in patients with asthma complicated by allergic rhinitis.

Although recent findings suggest allergic rhinitis as a possible therapeutic target for asthma control $[5,6]$, there are few reports available concerning the association of the responses to therapeutic agents for the two disorders. Here we had an opportunity to evaluate the clinical efficacies of leukotriene receptor antagonists (LTRAs) and anti-histamines in patients with asthma complicated by allergic rhinitis in practice.

\section{METHODS}

\section{Study design}

Consecutive patients with asthma with/without concomitant allergic rhinitis were recruited at 217 medical institutions in eight prefectures of the Kyushu district of Japan between May and December 2003 [6]. Recruited subjects in the study were non-smoking ambulatory adult patients being treated at one of the participating institutions who gave written consent to the survey. The survey consisted of systematic selfadministered questionnaires that were collected twice, one month apart, to obtain information on subjective symptoms, self-evaluated disease control and satisfaction with treatment. Physicians provided a complete list of medications for asthma and allergic rhinitis on both occasions. The questionnaire also included items for medications. The study protocol was conducted in accordance with the Declaration of Helsinki and approved by institutional board of ethical committees, and all participants gave written informed consent.

\section{Definitions of asthma and allergic rhinitis}

The diagnosis of asthma was based on the Global Initiative for Asthma guideline [7]. Patients were considered as having allergic rhinitis if they presented with sneeze or nasal itching, watery nasal discharge, and nasal obstruction, and either eosinophilia in nasal discharge or positive results for positive skin prick or serum specific IgE for common antigens (house dust and pollens).

\section{Self-administered questionnaire}

Patients completed the questionnaire by selecting one of the relevant ratings for each items [6]. Items related to allergic rhinitis included sneeze (0:none, 1:1 to 5, 2:more than 6 times a day), rhinorrhea (0:none,
1:1 to 5,2 : nose blowing more than 6 times a day), nasal obstruction (0:none, 1:mild, 2:moderate to severe), and over-all nasal symptoms (1:excellent, 2:good, 3 :worse than good). Items related to asthma included wheeze (0:none, 1:1 to 2, 2:more than 3 days a week for morning, daytime, nighttime separately), dyspnea (same rating as wheeze), cough (0:none, 1:1 to 2, 2:more than 3 days a week), sputum (same rating as cough), and over-all asthma symptoms (same rating as over-all nasal symptoms) and satisfaction with treatment (1:satisfied, 2: mostly satisfied, 3:other than 1 and 2).

\section{Statistical analysis}

Comparisons between the groups were performed by Mann-Whitney non-parametric U test or chi-square test where appropriate. Wilcoxon signed-rank test was used for the paired comparisons of the same individuals. All statistical analyses were performed using SPSS software 13.0J (Spss Japan Inc., Tokyo, Japan).

\section{RESULTS}

The questionnaire was distributed to 3,270 patients with asthma who gave consent to the survey, and was completed by 3,140 of them. Of these, 634 patients had physician-diagnosed allergic rhinitis in addition to asthma, including 19 patients who began receiving anti-histamines (loratadine in 6, ebastine in 4, fexofenadine in 2, olopatadine in 2, and azelastine, epinastine, emadastine, chlorpheniramine, mequitazine in 1 patient each) immediately after the first survey, without any other changes in medications, and 26 patients who began to receive LTRAs (pranlukast for 21 patients and montelukast for 5 patients) without any other treatment modifications during the same period. Demographics and treatments of these patients were summarized in Table 1. When these two groups of patients were compared, there were no significant differences in gender, age, base-line medications (inhaled, intranasal and oral corticosteroids and bronchodilators) or severity of asthma as judged by the medications.

Comparisons of the nasal and asthma symptoms between the two patient groups on the first survey (Table 2) revealed that there were significant differences in some of the symptoms related with asthma: patients who were treated with LTRAs had significantly more severe wheezes in the morning, daytime and nighttime, daytime dyspnea, cough, sleep disturbance, and were significantly less satisfied with the asthma treatment in comparison with the patients who received antihistamines. There were no significant dif- 
TABLE 1.

Patient characteristics

\begin{tabular}{cccc}
\hline & Anti-histamines & LTRAs & $p$ \\
\hline $\mathrm{n}$ & 19 & 26 & \\
Female (\%) & $8(42.1)$ & $11(42.3)$ & 0.62 \\
Age (SD) [year] & $50.2(19.2)$ & $46.2(19.9)$ & 0.78 \\
$\beta 2$ agonists* (\%) & $3(15.8)$ & $8(30.8)$ & 0.24 \\
Theophylline (\%) & $9(47.4)$ & $17(65.4)$ & 0.36 \\
INS (\%) & $4(21.1)$ & $5(19.2)$ & 1.0 \\
ICS (\%) & $18(94.7)$ & $19(73.1)$ & 0.06 \\
ICS dose (SD) [mg] & $412.5(185.7)$ & $450.0(225.1)$ & 0.52 \\
Oral steroid & $1(0.05)$ & $3(0.1)$ & 0.63 \\
\hline
\end{tabular}

*includes dermal patch and inhalation formula

LTRAs, leukotriene recptor antagonists; INS, intranasal corticosteroid; ICS, inhaled corticosteroid Paretheses indicate SD

TABLE 2.

Comparison of the symptoms on the first survey

\begin{tabular}{cccc}
\hline & \multicolumn{2}{c}{ Sum of the ranks } & \\
\cline { 2 - 3 } & Anti-histamies & LTRAs & 0.80 \\
Sneeze & 397 & 593 & 0.46 \\
Rhinorrhea & 330 & 660 & 0.18 \\
Nasal obstruction & 388 & 647 & 0.81 \\
Over all nasal & 392 & 553 & 0.14 \\
Limitation in daily activity & 336 & 610 & 0.009 \\
Morning wheeze & 314 & 677 & 0.058 \\
Morning dyspnea & 310 & 637 & 0.035 \\
Daytime wheeze & 323 & 623 & 0.049 \\
Daytime dyspnea & 335 & 611 & 0.022 \\
Nighttime wheeze & 334 & 656 & 0.352 \\
Nighttime dyspnea & 377 & 613 & 0.041 \\
Cough & 309 & 595 & 0.133 \\
Sputum & 348 & 598 & 0.001 \\
Sleep disturbance & 272 & 674 & 0.059 \\
Over all asthma & 365 & 670 & 0.025 \\
Satisfaction to treatment & 350 & 686 & \\
\hline
\end{tabular}

ferences in the initial symptoms related with rhinitis.

Comparisons of the nasal and asthma symptoms between the first and second questionnaire were summarized in Table 3. In nasal symptoms, there were no significant changes in the patients treated with antihistamines. In contrast, the survey results demonstrated that there were significant improvements in sneeze and rhinorrhea in patients treated with LTRAs. Nasal obstruction tended to be improved in patients treated with LTRAs, but not in those treated with anti-histamines.

There were no significant changes in any of asthmarelated symptoms in patients treated with anti-histamines, whereas there were significant improvements in morning dyspnea, sleep disturbance, over-all asthma symptoms, and satisfaction with asthma treatment in 
TABLE 3

Efficacies of anti-histamines and LTRAs

\begin{tabular}{|c|c|c|c|c|c|c|c|c|}
\hline & \multicolumn{4}{|c|}{ Anti-histamines } & \multicolumn{4}{|c|}{ LTRAs } \\
\hline & First & Second & $\mathrm{z}$ & $p$ & First & Second & $\mathrm{z}$ & $p$ \\
\hline Sneeze & $1.0(0.49)$ & $0.94(0.54)$ & -0.45 & 0.65 & $1.04(0.53)$ & $0.68(0.69)$ & -2.18 & 0.03 \\
\hline Rhinorrhea & $0.94(0.64)$ & $1.00(0.76)$ & -0.45 & 0.65 & $1.35(0.63)$ & $1.00(0.76)$ & -2.5 & 0.01 \\
\hline Nasal obstruction & $1.26(0.45)$ & $1.22(0.43)$ & -0.45 & 0.65 & $1.46(0.51)$ & $1.24(0.44)$ & -1.89 & 0.06 \\
\hline Over all nasal & $1.94(0.24)$ & $1.76(0.43)$ & -1.73 & 0.08 & $1.96(0.20)$ & $1.80(0.41)$ & -1.63 & 0.1 \\
\hline Limitation in daily activity & $0.06(0.24)$ & $0(0)$ & -1 & 0.32 & $0.23(0.43)$ & $0.16(0.37)$ & -0.71 & 0.48 \\
\hline Morning wheeze & $0.17(0.51)$ & $0.11(0.32)$ & -0.58 & 0.56 & $0.77(0.86)$ & $0.5(0.71)$ & -1.46 & 0.14 \\
\hline Morning dyspnea & $0.24(0.56)$ & $0.22(0.43)$ & 0 & 1 & $0.65(0.80)$ & $0.36(0.57)$ & -2 & 0.046 \\
\hline Daytime wheeze & $0(0)$ & $0(0)$ & 0 & 1 & $0.31(0.62)$ & $0.35(0.56)$ & -0.33 & 0.73 \\
\hline Daytime dyspnea & $0.11(0.32)$ & $0(0)$ & -1.41 & 0.16 & $0.56(0.82)$ & $0.42(0.75)$ & -1 & 0.32 \\
\hline Nighttime wheeze & $0.06(0.23)$ & $0.12(0.33)$ & -0.57 & 0.56 & $0.54(0.81)$ & $0.38(0.64)$ & -1.26 & 0.2 \\
\hline Nighttime dyspnea & $0.17(0.38)$ & $0.18(0.39)$ & 0 & 1 & $0.38(0.7)$ & $0.38(0.7)$ & -0.09 & 0.93 \\
\hline Cough & $0.06(0.24)$ & $0(0)$ & -1 & 0.32 & $0.44(0.71)$ & $0.26(0.67)$ & -1.02 & 0.3 \\
\hline Sputum & $0.22(0.55)$ & $0.16(0.37)$ & -0.58 & 0.56 & $0.6(0.87)$ & $0.38(0.7)$ & -1.73 & 0.08 \\
\hline Sleep disturbance & $0(0)$ & $0.11(0.32)$ & -1.41 & 0.16 & $0.65(0.8)$ & $0.25(0.61)$ & -2.33 & 0.02 \\
\hline Over all asthma & $1.79(0.42)$ & $1.67(0.49)$ & -1 & 0.32 & $2.15(0.73)$ & $1.81(0.57)$ & -2.5 & 0.013 \\
\hline Satisfaction to treatment & $1.32(0.58)$ & $1.18(0.39)$ & -1 & 0.32 & $1.85(0.83)$ & $1.46(0.65)$ & -2.35 & 0.019 \\
\hline
\end{tabular}

LTRAs, leukotriene receptor antagonists

Parentheses indicate SD.

those treated with LTRAs. Sputum tended to be improved in patients treated with LTRAs, but not in those treated with anti-histamines.

\section{DISCUSSION}

The present study focused on the clinical efficacy of anti-histamines and LTRAs in patients with asthma complicated by allergic rhinitis, and our results showed that the initiation of LTRAs but not anti-histamines was associated with significant improvement in asthmarelated symptoms. Published reports indicate that appropriate management of concomitant allergic rhinitis confers a better control of asthma in patients with both diseases. For instance, treatment with intranasal corticosteroid for allergic rhinitis resulted in decreased cough and severity of concomitant asthma [8]. A retrospective cohort study has documented that treatment of concomitant allergic rhinitis was associated with reductions in emergency visits or hospitalizations caused by asthma exacerbations [9]. In this context, anti-histamines and LTRAs, which are both commonly prescribed drugs, deserve attention as therapeutic agents not only for allergic rhinitis itself but also to achieve better control of concomitant asthma in patients with both diseases. A randomized study involving patients with asthma complicated by allergic rhinitis has documented that subjects treated with cetirizine, an anti-histamine, showed significant improvements in nasal and asthma symptoms, although pulmonary function was comparable with those who received a placebo [10], whereas another study has reported that the administration of cetirizine resulted in a significant improvement in lung function in subjects treated with the drug in comparison with the subjects who received placebo in patients with mild-to-moderate asthma [11]. In contrast, the present study did not support the efficacies of anti-histamines during the study period. Reasons for this discrepancy are unknown, however, several plausible explanations deserve mentioning. The first survey indicated that patients treated with LTRAs generally had had worse symptoms related with rhinitis and asthma, some of which were statistically significant, in comparison with patients treated with anti-histamines. It is possible that the difference in the severity of initial symptoms contributed to the observed difference noted in the second survey. Second, several varieties of anti-histamines were used 
in the present study, while only two kinds of LTRAs were prescribed, probably reflecting the difference in the number of available drugs. Since there is a significant variation in potency among anti-histamines [12], some of the anti-histamines or the doses used may not have been adequate to produce clinical improvements in our limited study period. Duration of treatment is another factor that may have affected the observed results. For instance, a significant reduction in bronchial hyperreactivity has been observed in patients treated with cetirizine for two weeks [13], whereas montelukast, a LTRA, showed its effectiveness in a shorter period in patients with asthma [14]. This may also explain the insignificant improvements in the nasal symptoms in patients treated with anti-histamines.

The results of the present study have demonstrated that some nasal and asthma symptoms improved significantly in patients treated with LTRAs. Interestingly, satisfaction with asthma treatment was also significantly improved in patients who started LTRAs. Therapeutic effects of LTRAs on asthma itself [15] and, as aforementioned, on nasal symptoms are both likely to have contributed to the improvement in patient satisfaction. A meta-analysis indicated that the efficacies of LTRAs are comparable with those of anti-histamines but less than those of nasal corticosteroids in patients with seasonal allergic rhinitis [16]. It would be interesting to compare the clinical efficacies of LTRAs and nasal corticosteroids as a treatment option in the management of patients with asthma complicating allergic rhinitis.

The primary limitation of the present investigation arises from its cross-sectional uncontrolled observational design, which may harbor a potential bias in the subjects for comparisons, although they were recruited consecutively from multiple institutions. A second limitation is the fact that the results solely depended on subjective evaluations of the symptoms and were not endorsed by objective parameters. In addition, limited information on medications hindered a detailed assessment of the efficacies of the drugs. Despite these limitations, however, the present investigation provided a unique opportunity to evaluate the efficacies of therapeutic agents in daily practice, and supported the intimate association between allergic rhinitis and asthma from a therapeutic viewpoint. Importantly, the results of the present investigation suggest a considerable difference in the therapeutic efficacy of medications used in the management of asthma complicating allergic rhinitis. Since the two diseases often co-exist, optimization of currently available treatments is needed to improve quality of care for affected individuals.

\section{REFERENCES}

1. Matsuno O, Miyazaki E, Takenaka R, Ando M, Ito T, et al. Links between bronchial asthma and allergic rhinitis in the Oita Prefecture, Japan. J Asthma 2006; 43:165-167.

2. Bousquet J, Boushey HA, Busse WW, Canonica GW, Durham SR et al. Characteristics of patients with seasonal allergic rhinitis and concomitant asthma. Clin Exp Allergy 2004; 34:897-903.

3. Ciprandi G, Cirillo I, Vizzaccaro A, Milanese M, and Tosca MA. Airway function and nasal inflammation in seasonal allergic rhinitis and asthma. Clin Exp Allergy 2004; 34:891-896.

4. Bonay M, Neukirch C, Grandsaigne M, Lecon-Malas V, Ravaud $\mathrm{P}$ et al. Changes in airway inflammation following nasal allergic challenge in patients with seasonal rhinitis. Allergy 2006; 61:111-118.

5. Bousquet J, Bullinger M, Fayol C, Marquis P, Valentin B et al. Assessment of quality of life in patients with perennial allergic rhinitis with the French version of the SF-36 Health Status Questionnaire. J Allergy Clin Immunol 1994; 94:182-188.

6. Koga T, Matsuse H, Kohrogi H, Kohno S and Aizawa H. Impact of nasal condition on self-assessed disease control and treatment satisfaction in patients with asthma complicated by allergic rhinitis. Allergol Int 2007; 56:427-431.

7. Global Initiative for Asthma (GINA). Global strategy for asthma management and prevention, 2002 Revision. Available from www.ginasthma.org

8. Henriksen JM and Wenzel A. Effect of an intranasally administered corticosteroid (budesonide) on nasal obstruction, mouth breathing, and asthma. Am Rev Respir Dis 1984; 130:1014-1018.

9. Crystal-Peters J, Neslusan C, Crown WH and Torres A. Treating allergic rhinitis in patients with comorbid asthma: the risk of asthma-related hospitalizations and emergency department visits. J Allergy Clin Immunol. 2002; 109:57-62.

10. Grant JA, Nicodemus CF, Findlay SR, Glovsky MM, Grossman J et al. Cetirizine in patients with seasonal rhinitis and concomitant asthma: prospective, randomized, placebo-controlled trial. J Allergy Clin Immunol 1995; 95:923-932.

11. Spector SL, Nicodemus CF, Corren J, Schanker HM, Rachelefsky GS et al. Comparison of the bronchodilatory effects of cetirizine, albuterol, and both together versus placebo in patients with mild-to-moderate asthma. J Allergy Clin Immunol 1995; 96:174-181.

12. Howarth PH, Stern MA, Roi L, Reynolds R and Bousquet J. Double-blind, placebo-controlled study comparing the efficacy and safety of fexofenadine hydrochloride (120 and $180 \mathrm{mg}$ once daily) and cetirizine in seasonal allergic rhinitis. J Allergy Clin Immunol 1999; 104:927-933.

13. Aubier M, Neukirch C, Peiffer C and Melac M. Effect of cetirizine on bronchial hyperresponsiveness in patients with seasonal allergic rhinitis and asthma. Allergy 2001; $56: 35-42$.

14. Malmstrom K, Rodriguez-Gomez G, Guerra J, Villaran C, Pineiro A et al. Oral montelukast, inhaled beclomethasone, and placebo for chronic asthma. A randomized, controlled 
trial. Montelukast/Beclomethasone Study Group. Ann Intern Med 1999; 1306:487-495.

15. Currie GP and Lipworth BJ. Bronchoprotective effects of leukotriene receptor antagonists in asthma: a meta-analysis. Chest 2002; 122:146-150.
16. Wilson AM, O'Byrne PM and Parameswaran K. Leukotriene receptor antagonists for allergic rhinitis: a systematic review and meta-analysis. Am J Med 2004; 116:338-344. 